\title{
Investigations on the magnetic field coupling of automotive high voltage systems to determine relevant parameters for an EMR-optimized designing
}

\author{
David Krause $^{1}$, Werner John ${ }^{2}$, and Robert Weigel ${ }^{3}$ \\ ${ }^{1}$ AUDI AG, 85045 Ingolstadt, Germany \\ ${ }^{2}$ SiL System Integration Laboratory GmbH, Technologiepark 32, 33100 Paderborn, Germany \\ ${ }^{3}$ Lehrstuhl für Technische Elektronik, Friedrich-Alexander-Universität Erlangen-Nürnberg, \\ Cauerstraße 9, 91058 Erlangen, Germany
}

Correspondence to: David Krause (david.krause@audi.de)

Received: 16 January 2015 - Revised: 14 December 2015 - Accepted: 11 March 2016 - Published: 21 March 2016

\begin{abstract}
The implementation of electrical drive trains in modern vehicles is a new challenge for EMC development. This contribution depicts a variety of investigations on magnetic field coupling of automotive high-voltage (HV) systems in order to fulfil the requirements of an EMR-optimized designing. The theoretical background is discussed within the scope of current analysis, including the determination of current paths and spectral behaviour. It furthermore presents models of shielded HV cables with particular focus on the magnetic shielding efficiency. Derived findings are validated by experimental measurements of a state-of-the-art demonstrator on system level. Finally EMC design rules are discussed in the context of minimized magnetic fields.
\end{abstract}

\section{Introduction}

The development of EMC concepts for electric vehicles requires a thorough understanding of electromagnetic coupling mechanisms of the high-voltage (HV) system. Due to the limited freedom of modification of vehicle drive-systems, available resources needs to be used optimally, which further demands identification and evaluation of EMC-related design parameters. Comparing the wavelengths of dominant electromagnetic interferences from voltage source inverters (VSI) with the geometrical dimension of a regularly sized vehicle enables a quasi-steady approach, where electric and magnetic fields can be investigated separately. In this con- tribution the focus is laid on the magnetic field coupling of automotive HV systems.

The pulse-wide modulated voltage switching of power electronics generates parasitic currents apart from operational ones. They are primary caused by distributed capacitances present in $\mathrm{HV}$ components like the electric motor. In general current levels are scaling with the size of the components, because larger conductive structures mean an increased capacitive coupling. In other words, engines with more electric power generate more parasitic current that consequently causes higher magnetic fields. Apart from the current strength, magnetic field coupling on antennas, wires or other electric devices is significantly dependent on the geometry of cable routes and the characteristics in frequency domain. Related investigations therefore require identifying major current paths as well as the respective spectral behaviour, which had been the focus of former contributions e.g. Kempski et al. (2006), Grandi et al. (2004) and Jettanasen (2010), but were not considered in the context of magnetic fields.

Research work presents selected experimental EMI measurements of a state-of-the-art HV system demonstrator, focusing on quasi-steady magnetic fields with frequencies from $9 \mathrm{kHz}$ until the lower $\mathrm{MHz}$ range. The theoretical background includes a current analysis of the AC-network, where common-mode (CM) and differential-mode (CM) circuit models are used to determine the underlying spectral functions. As fully shielded systems are commonly used in automotive HV systems (Hohloch et al., 2012), shielded HV ca- 
ble models are introduced and used to analytically describe the magnetic shielding efficiency. In a further step they are verified by numerical field simulations and measurements.

With the help of a state-of-the-art system demonstrator derived findings are experimentally proved. Loop-antenna measurements are analysed in the scope of developed circuit models and spectral functions. It is demonstrated, that different operating conditions of the VSI can show either CM- or DM dominant magnetic coupling. Slight adjustments at the test setup made it possible to investigate unshielded and shielded conditions with reference to EMCrequirements (GB/T 18387, 2008), which are relevant for the type-approval of electric vehicles. Finally this contribution concludes with a discussion about EMC design rules and intents to support electric power train layout designers in dimensioning EMC-optimized HV topologies.

\section{Current analysis}

The application of VSIs into electric vehicles leads to a broadband spectrum of challenging EMC issues. One important aspect, discussed in this chapter, is the investigation of currents with their respective propagation paths and spectral functions. Based on the requirements of automotive EMC norms, which are defined particularly for electric vehicles, the relevant frequencies are ranging from $9 \mathrm{kHz}$ until $30 \mathrm{MHz}$ (GB/T 18387, 2008).

Due to reasons of personal safety automotive HV systems are always built up as IT(isole terra)-networks and are therefore isolated from the vehicle chassis, representing the common ground system. This consideration is only valid from the DC perspective and needs to be reversed, when investigating higher frequencies. From the EMC point of view the system then becomes more complex and parasitic properties (e.g. stray capacitances) need to be minded (see Fig. 1).

The basic task of the VSI is to generate changeable rotational speed for the electric engine by offering sinusoidal pulse-modulated voltages. These periodic voltage pulses are causing currents, which can be represented by a series of harmonics in frequency domain. For EMC analyses in general it is useful to distinguish between common-mode (CM) and differential-mode (DM). First one is describing the electrical behaviour to the ground system that in terms of automotive HV systems primary has a capacitive nature. Hence, time varying voltages like pulses lead to a flow of displacement current proportional to the value of systemic parasitical capacitances:

$i_{\mathrm{C}}(t)=C \frac{\mathrm{d} u_{\mathrm{C}}(t)}{\mathrm{d} t}$.

The second one, representing the symmetrical line-to-line behaviour, additionally shows major inductive influence especially for the very low frequency range. Current then is caused by the integration of the time-varying voltage pulses and is indirectly proportional to the inductance value:

$i_{\mathrm{L}}(t)=\frac{1}{L} \int u_{\mathrm{L}}(t) \mathrm{d} t$.

The asymmetrical control of inverters leads to both CM and DM voltages and thus forms respective current paths with their own spectral characteristics that will be in detail discussed with focus on the AC-network below. Derivated linear equations are using variable spectral functions and are therefore independent from the underlying modulation technique. However, in the literature there are analytical closedform expressions for all modulation methods, which are currently applied in automotive power electronics (SVPWM and Flat Top). The most native method, the sine-triangular PWM or SPWM, is used for the following SPICE-based calculations and for example can be analytically represented by Double Fourier analysis as a sum of carrier and side-band harmonics (Kempski et al., 2006).

\subsection{Common-Mode current}

CM currents, commonly also known as leakage currents, are always parasitical and therefore not part of the actual function of electric power trains. They are generated by alternating voltage drops at capacitively coupled reference systems that in automotive terms are not restricted to vehicle chassis, but also can be low-voltage (LV) networks or large conductive structures like motor shafts. In this contribution the basic $\mathrm{CM}$ behaviour of the AC-network is described, where some simplifications are performed.

For IT-based HV systems the CM path is significantly determined by its capacitive properties and is therefore modelled by discrete Y-capacitances (see Fig. 2). In real application they are distributed and shaped primary between the stator and the housing on the motor side and between the IGBT modules and the metallic cooling unit on the inverter side (Kempski et al., 2002). Inductances are mainly determined by $\mathrm{HV}$ cables, whereas for $\mathrm{CM}$ considerations the inductive influence of the motor coils can be neglected, because of bypassing winding capacitances. Since oscillating circuits are formed, the model additionally needs to include DC-resistances to avoid unrealistically sharp resonances.

The EMC-relevant CM source is the switched voltage drop between the phase- and the reference system. For a threephase inverter the respective voltage is given by the vector sum of the instantaneous pulsed phase voltages (e.g., Cacciato et al., 1999),

$\underline{U}_{\mathrm{CM}}=\frac{\underline{U}_{u, \mathrm{gr}}+\underline{U}_{v, \mathrm{gr}}+\underline{U}_{w, \mathrm{gr}}}{3}$

which is never zero, because of temporary electrical asymmetry (Kempski et al., 2002). According to the circuit, there is a characteristic resonance frequency at:

$\omega_{\mathrm{CM}, r}=\frac{1}{\sqrt{\frac{C_{\mathrm{Y}, \mathrm{vsi}} \cdot C_{\mathrm{Y}, \mathrm{mot}}}{C_{\mathrm{Y}, \mathrm{vsi}}+C_{\mathrm{Y}, \mathrm{mot}}} \cdot \frac{L_{\mathrm{wr}}}{3}}}$. 


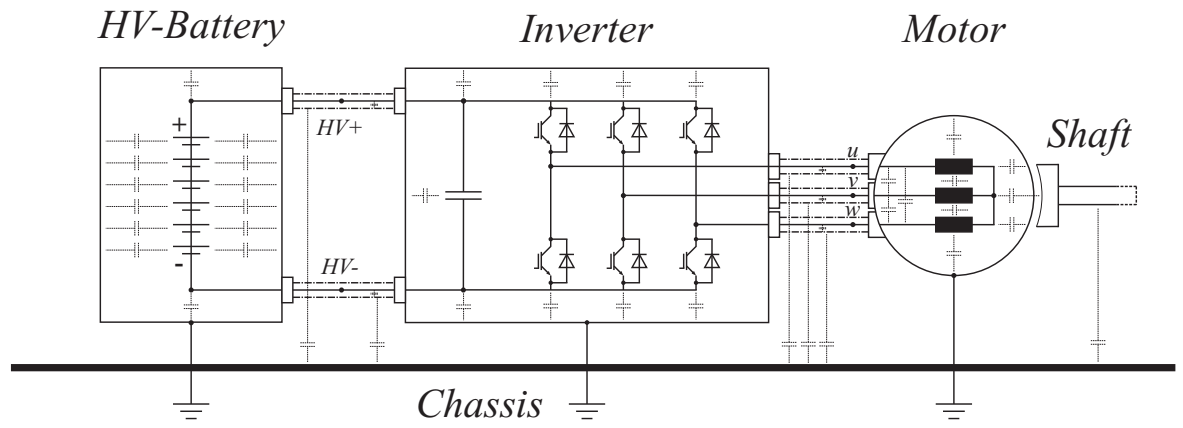

Figure 1. Distributed parasitic capacitances in automotive HV systems.

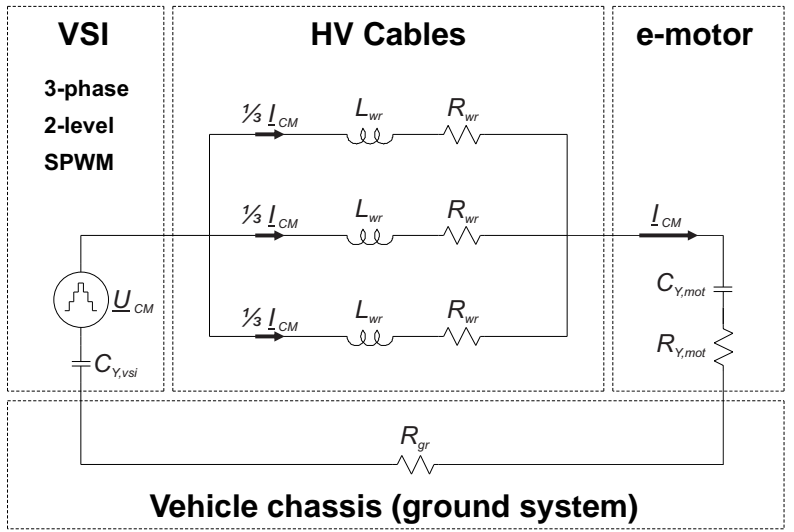

Figure 2. Simplified CM circuit model of the AC-/phase-network.

Hence, the spectral function of the CM current can be piecewise defined with:

$$
\underline{I}_{\mathrm{CM}}= \begin{cases}\underline{U}_{\mathrm{CM}} \cdot \frac{j \omega C_{\mathrm{Y}, \mathrm{mot}} \cdot C_{\mathrm{Y}, \mathrm{vsi}}}{C_{\mathrm{Y}, \mathrm{vsi}}+C_{\mathrm{Y}, \mathrm{mot}}} & \text { if } \omega \ll \omega_{\mathrm{CM}, r} \\ \underline{U}_{\mathrm{CM}} \cdot \frac{1}{R_{\mathrm{wr}} / 3+R_{\mathrm{Y}, \mathrm{mot}}} & \text { if } \omega=\omega_{\mathrm{CM}, r} \\ \underline{U}_{\mathrm{CM}} \cdot \frac{3}{j \omega L_{\mathrm{wr}}} & \text { if } \omega \gg \omega_{\mathrm{CM}, r}\end{cases}
$$

Typical CM parameters are summarized in Table 1 and used to calculate the circuit model with SPICE (see Fig. 3). In time domain the voltage pulses are formed as step functions with three positive and negative steps. During the slopes, capacitances are excited and leakage current peaks are generated. According to the inverter's clock frequency pulses are periodically repeated, that consequently leads to an integer multiple of clock harmonics in frequency domain. Besides, there are additional side band harmonics, following the fundamental frequency of the modulated sine waves. For the observed frequency range until $30 \mathrm{MHz}$, the voltage source offers rectangular signals, hence harmonic levels are constantly decreasing with $20 \mathrm{~dB}$ per decade. Since Y-capacitances of VSIs are typically much higher than those of electric en-
Table 1. Relevant parameters for calculating CM voltages and currents.

\begin{tabular}{lll}
\hline Parameter & Description & Value \\
\hline$U_{\mathrm{DC}}$ & DC-voltage VSI & $300 \mathrm{~V}$ \\
$f_{\mathrm{c}}$ & Clock frequency VSI & $10 \mathrm{kHz}$ \\
$M$ & Modulation index SPWM & $50 \%$ \\
$R_{\mathrm{wr}}, R_{\mathrm{gr}}$ & Resistance HV cable, ground & $0.5 \mathrm{~m} \Omega$ \\
$L_{\mathrm{wr}}$ & Self-inductance 2 m HV cable & $2 \mu \mathrm{H}$ \\
$R_{\mathrm{Y}, \mathrm{mot}}$ & Y-path resistance e-Motor & $3 \Omega$ \\
$C_{\mathrm{Y}, \mathrm{mot}}$ & Y-path capacitance e-Motor & $6 \mathrm{nF}$ \\
$C_{\mathrm{Y}, \mathrm{vsi}}$ & Y-path capacitance VSI & $100 \mathrm{nF}$ \\
\hline
\end{tabular}

gines, the current level is mainly restricted by the capacitive coupling at the motor side (see Eq. 5). According to Eq. (4) there is a resonance point at approximately $2.5 \mathrm{MHz}$, where the reactance is zero and the current level is determined by the overall DC resistance. The current spectrum behaves flat for frequencies far below, since simultaneously harmonics of the pulsed voltage are decreasing and the admittance of the CM path is rising with $20 \mathrm{~dB}$ per decade. Following again Eq. (5) the CM impedance for the clock frequency at $10 \mathrm{kHz}$ equals $69 \mathrm{~dB} \Omega$, which expectably is the same result as the difference of voltage and current levels in the logarithmic frequency domain plot. The self-inductances of the HV cables become decisive, when frequencies far above the resonance point are considered. Here, currents are well attenuated and therefore less relevant from EMC perspective.

\subsection{Differential-Mode current}

When referring to DM currents of automotive HV systems, the following separation is useful:

- operational load currents

- current ripples

- capacitive displacement currents

Operational load currents represent the $120^{\circ}$ phase shifted rotary current at the motor coils. Frequencies are equal to the 


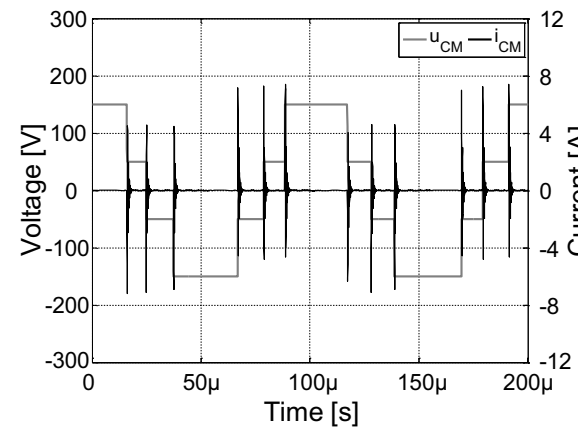

Figure 3. CM voltage and current in time and frequency domain.

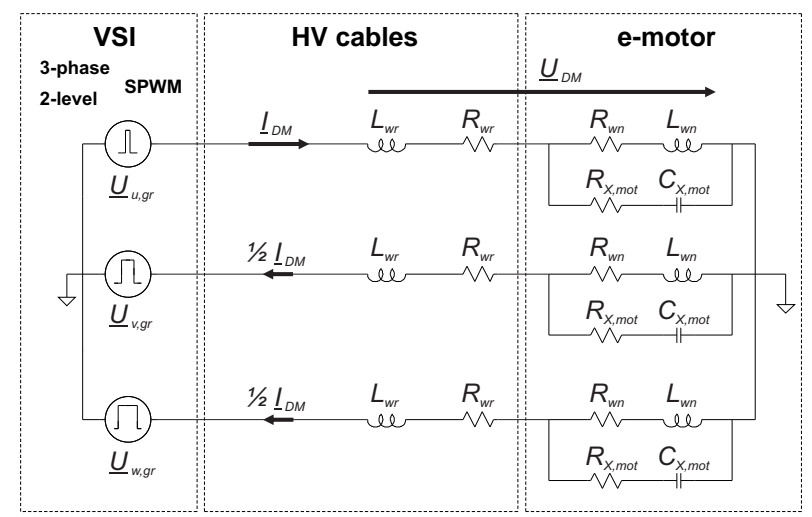

Figure 4. DM circuit model of the AC-network.

fundamental component of the respective modulation technique and in automotive applications are typically not higher than $1 \mathrm{kHz}$. As a functional part of the electric power train these currents will not be investigated, since their properties are outside the boundary conditions of this contribution. Current ripples and capacitive displacement currents contrarily lie in a relevant frequency area, have parasitic origins and are going to be discussed in this section.

To analyse them for a three-phase VSI, a symmetrical circuit model is used, where the electric machine is represented by ohmic-inductive loads in star connection (see. Fig. 4).

Parasitic properties are modelled by parallel Xcapacitances, which are primary formed at the coil windings and the connector panel. The effective DM source is an arbitrary phase leg voltage given by:

$\underline{U}_{\mathrm{DM}}=\underline{U}_{u, \mathrm{gr}}-\underline{U}_{\mathrm{CM}}=\frac{2 \cdot \underline{U}_{u, \mathrm{gr}}-\underline{U}_{v, \mathrm{gr}}+\underline{U}_{w, \mathrm{gr}}}{3}$

According to the circuit model there are two characteristic frequencies. The first one is defined by the parallel circuit resonance of self-inductances and parallel X-capacitances, where the motor impedance changes from inductive to capacitive behaviour.

$\omega_{\mathrm{DM}, \mathrm{r} 1}=\frac{1}{\sqrt{C_{\mathrm{X}, \mathrm{mot}} \cdot L_{\mathrm{wn}}}}$

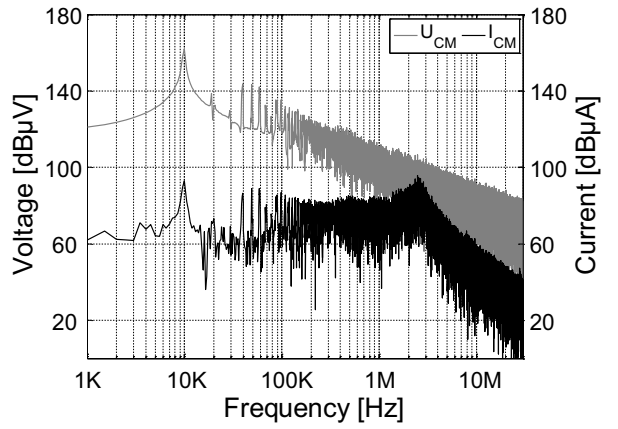

The second one is formed by the series circuit of wire inductances and X-capacitances and is simultaneously defining a resonance point of the overall DM path:

$\omega_{\mathrm{DM}, \mathrm{r} 2}=\frac{1}{\sqrt{C_{\mathrm{X}, \mathrm{mot}} \cdot L_{\mathrm{wr}}}}$

Assuming reasonable circumstances, where $L_{\mathrm{wn}}>L_{\mathrm{wr}}$ and consequently $\omega_{\mathrm{DM}, \mathrm{r} 1}<\omega_{\mathrm{DM}, \mathrm{r} 2}$, DM currents can be piecewise defined with:

$$
\underline{I}_{\mathrm{DM}}= \begin{cases}\underline{U}_{\mathrm{DM}} \cdot \frac{1}{R_{\mathrm{wn}}+j \omega L_{\mathrm{wn}}} & \text { if } \omega \ll \omega_{\mathrm{DM}, \mathrm{r} 1} \\ \underline{U}_{\mathrm{DM}} \cdot j \omega C_{\mathrm{X}, \mathrm{mot}} & \text { if } \omega_{\mathrm{DM}, \mathrm{r} 1 \ll \omega \ll \omega_{\mathrm{DM}, \mathrm{r} 2}} \\ \underline{U}_{\mathrm{DM}} \cdot \frac{1}{R_{\mathrm{wr}}+R_{\mathrm{x}, \mathrm{mot}}} & \text { if } \omega=\omega_{\mathrm{DM}, \mathrm{r} 2} \\ \underline{U}_{\mathrm{DM}} \cdot \frac{1}{j \omega L_{\mathrm{wr}}} & \text { if } \omega \gg \omega_{\mathrm{DM}, \mathrm{r} 2}\end{cases}
$$

For typical DM parameters summarized in Table 2 respective voltages and currents are calculated with SPICE (see Fig. 5). Again periodically pulsed voltages are present that can be represented by Fourier series representations as a set of harmonics starting from the clock frequency. For frequencies below the first edge frequency $\omega_{\mathrm{DM}, \mathrm{r} 1}$ the impedance is still ohmic-inductive (see Eq. 9) and currents are attenuated between 20 and $40 \mathrm{~dB}$ decade $^{-1}$. In time domain they can be identified as current ripples, superpositioning the operational sine currents. Considering even higher frequencies capacitive displacement currents outbalance current ripples, because of parasitic X-capacitances parallel to the motor coils. From now on the current spectrum behaves equally to the $\mathrm{CM}$ case with a resonance point at $\omega_{\mathrm{DM}, \mathrm{r} 2}$.

\section{Magnetic field coupling of shielded HV cables}

Nowadays automotive HV systems used in series electric vehicles are fully shielded (Hohloch et al., 2012). In addition 

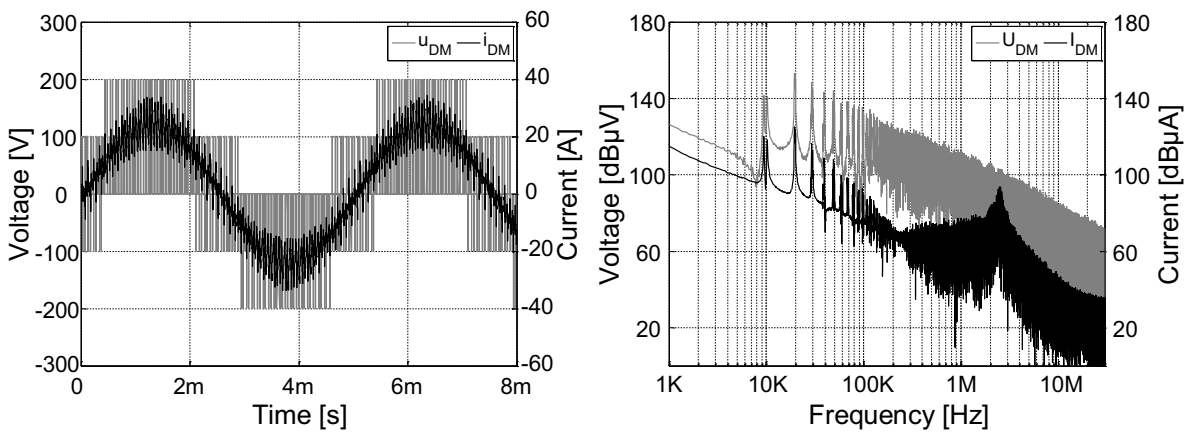

Figure 5. DM voltage and current in time and frequency domain.

Table 2. Relevant parameters for calculating DM voltages and currents.

\begin{tabular}{lll}
\hline Parameter & Description & Value \\
\hline$U_{\mathrm{DC}}$ & DC-voltage VSI & $300 \mathrm{~V}$ \\
$f_{\mathrm{c}}$ & Clock frequency VSI & $10 \mathrm{kHz}$ \\
$f_{i}$ & Fundamental frequency VSI & $200 \mathrm{~Hz}$ \\
$M$ & Modulation Index SPWM & $50 \%$ \\
$R_{\mathrm{wr}}$ & Resistance HV cable & $1 \mathrm{~m} \Omega$ \\
$L_{\mathrm{wr}}$ & Self-inductance HV cable & $0.5 \mu \mathrm{H}$ \\
$R_{\mathrm{X}, \mathrm{mot}}$ & X-path resistance e-Motor & $3 \Omega$ \\
$C_{\mathrm{X}, \mathrm{mot}}$ & X-path capacitance e-Motor & $2 \mathrm{nF}$ \\
$R_{\mathrm{wn}}$ & Resistance coil windings & $3 \Omega$ \\
$L_{\mathrm{wn}}$ & Self-inductance coil windings & $200 \mu \mathrm{H}$ \\
\hline
\end{tabular}

to the metallic enclosures of HV components it implies, that also cable screens ensure a complete conductive surrounding of parts under (high) voltage. According to Ampère's law, an arbitrary closed-loop line integral of the magnetic field surrounding a shielded cable equals the vectorial sum of its inner wire current $\underline{I}_{\text {in }}$ and shield current $\underline{I}_{\mathrm{sh}}$ :

$\oint_{C} \underline{\boldsymbol{H}} \mathrm{d} s=\underline{I}_{\text {in }}+\underline{I}_{\mathrm{sh}}=\underline{I}_{\mathrm{sum}}$

For coaxial cables used in telecommunication systems, it is assumed that $\underline{I}_{\text {in }}=-\underline{I}_{\text {sh }}$ and the outer magnetic field is completely compensated. However, in the scope of power electronics applications this condition is not fulfilled, since operation modes of the cables as well as the considered frequency range are significantly different. Here, the residual sum current $\underline{I}_{\text {sum }}$ is causing an EMC-relevant magnetic field. In case of single shielded wire, which is routed straight and far away from its ground plane, the magnitude of the magnetic field vector can be calculated with the common formula:

$\underline{H}(r)=\frac{\underline{I}_{\text {sum }}}{2 \pi \cdot r}$

In the following subsections this basic approach is used to find analytical expressions for calculating the magnetic shielding efficiency (SE) of HV cables based on equivalent circuit models. In a further step they are validated by numerical field simulation and laboratory measurements.

\subsection{Shielded cable models for quasi-steady currents}

As shown in Sect. 2 the significant spectral content of currents is ranging until several Megahertz. In this context shielded HV cables can be modeled by their ohmic and inductive behaviour and capacitive displacement currents are neglectable (see Fig. 6).

Shielding against quasi-steady magnetic fields is dominantly driven by the mutual inductance between inner wire and the shield. Induced voltages lead to shield currents, that are dependent on the screen impedance and cause compensating fields (see Eq. 10). For the CM case the ground system also needs to be included. In contrast to the cables it shows minor inductive influence and is therefore modelled as a resistive path. Since pure symmetrical conditions are defined for the DM case, a potential ground path would carry no current and is thus not considered.

\subsection{Calculating the magnetic shielding efficiency}

Remembering the above mentioned definition, where the sum current $\underline{I}_{\text {sum }}$ is directly proportional to the outer magnetic field (see Eq. 11), the magnetic SE can be calculated with the following expressions:

$$
\begin{aligned}
\mathrm{SE}_{H} & =20 \log _{10}\left|\frac{\underline{I}_{\text {in }}}{\underline{I}_{\text {sum }}}\right|=20 \log _{10}\left|\frac{\underline{I}_{\text {in }}}{\underline{I}_{\text {in }}+\underline{I}_{\text {sh }}}\right| \\
& =-20 \log _{10}\left|1+\frac{\underline{I}_{\text {sh }}}{\underline{I}_{\text {in }}}\right|
\end{aligned}
$$

According to the CM circuit model (Fig. 6, left) the current ratio between cable shield and inner wire is given by:

$$
\frac{\underline{I}_{\mathrm{CM}, \mathrm{sh}}}{\underline{I}_{\mathrm{CM}, \mathrm{in}}}=-\frac{R_{\mathrm{CM}, \mathrm{gr}}}{R_{\mathrm{CM}, \mathrm{gr}}+R_{\mathrm{CM}, \mathrm{sh}}} \cdot \frac{1+j \omega \frac{M_{\mathrm{CM}}}{R_{\mathrm{CM}, \mathrm{gr}}}}{1+j \omega \frac{L_{\mathrm{CM}, \mathrm{sh}}}{R_{\mathrm{CM}, \mathrm{gr}}+R_{\mathrm{CM}, \mathrm{sh}}}}
$$

This formula is likewise valid for a multi-conductor system, when each of the wire has the same properties and its 


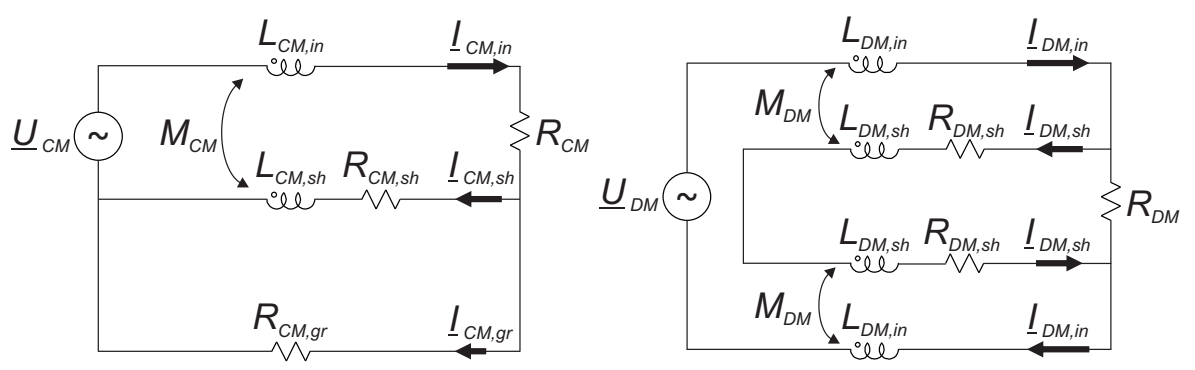

Figure 6. Simplified circuit models of shielded HV cables (left: CM; right: DM).

own ground path. In case $n$ conductors are sharing a single ground path, the DC-resistance needs to be reduced by $R_{\mathrm{gr}} / n$.

Now considering a DM voltage source feeding two or more conductors (see Fig. 6, right). Since pure symmetrical conditions are assumed, ground currents compensate and the ground path can be discarded. Setting $R_{\mathrm{gr}} \rightarrow 0$ into Eq. (13) leads to the DM current ratio

$\frac{\underline{I} \mathrm{DM}, \mathrm{sh}}{I_{\mathrm{DM}, \mathrm{in}}}=-\frac{j \omega M_{\mathrm{DM}}}{R_{\mathrm{DM}, \mathrm{sh}}+j \omega L_{\mathrm{DM}, \mathrm{sh}}}$.

In the literature there are closed analytical expressions to calculate the necessary inductances (Paul, 2006). For wires, parallel to a perfectly conducting wall (CM), the l.p.u. inductances is given by

$L_{\mathrm{CM}, \mathrm{in}}=\frac{\mu_{0}}{2 \pi} \cdot \ln \left(\frac{2 d}{a}\right)$,

where $a$ is the inner wire radius and $d$ is the distance of current carrying conductors. Analogously the 1.p.u. inductance of a single wire, as part of two parallel symmetrical wires (DM), can be calculated with:

$L_{\mathrm{DM}, \mathrm{in}}=\frac{\mu_{0}}{2 \pi} \cdot \ln \left(\frac{d}{a}\right)$

Because current paths of inner wires and shields are approximately the same, $L_{\mathrm{in}} \approx L_{\mathrm{sh}} \approx M$ can be assumed.

\subsection{Validation with simulations and measurements}

In the following numerical simulations and laboratory measurements (see Fig. 7) are performed to verify the calculation method presented in the previous section.

The CM setup is a one-cable-configuration, which is located $50 \mathrm{~mm}$ above a ground plane. Both, the source and the load are positioned between the inner wire and the ground potential. DM simulation and measurement setups are built up as symmetrical two-cable-configurations with an intercable distance of $50 \mathrm{~mm}$. The current is set to flow inbetween the wires, where one cable carries the load and the other one the return current. Since pure symmetrical conditions are ensured, the ground plane carries no current and can either be connected or isolated. To allow a flow of shield current, cable screens are connected at both ends to conductive structures and thus sharing their potentials with the ground plane. Since the magnetic SE is independent of the current strength and the load impedance, both are arbitrarily chosen. The resistance of the overall shield path is $2 \mathrm{~m} \Omega$, including the intrinsic screen resistance and transfer resistances of the shield connections. The ground path has only a minor resistive influence of $0.1 \mathrm{~m} \Omega$, which is the general case for automotive applications.

Numerical field simulations are performed by the LF Frequency Domain Solver of Computer Simulation Technology (CST) EM Studio (CST EM Studio Manual, 2014), based on the finite integration technique (FIT). Using the magnetoquasistatic (MQS) condition, where displacement currents are discarded, helps to significantly accelerate simulation speed and accuracy. At an arbitrary point $P\left(x_{0}, y_{0}, z_{0}\right)$, outside the shielded cable, the absolute value of the magnetic field vector is calculated for shielded and unshielded conditions. Hence, the magnetic SE is determined with:

$\mathrm{SE}_{\mathrm{H}, \mathrm{sim}}=20 \log _{10}\left|\frac{\left|\underline{\boldsymbol{H}}_{\mathrm{ush}}\left(x_{0}, y_{0}, z_{0}\right)\right|}{\left|\underline{\boldsymbol{H}}_{\mathrm{sh}}\left(x_{0}, y_{0}, z_{0}\right)\right|}\right|$

A VNA based measurement method was introduced by Feldhues et al. (2014) to investigate the current distribution of shielded HV cables for low frequency magnetic fields. In this context the same method is used to perform measurements of the magnetic SE. Port 1 feeds the cable configurations with impressed current. A current clamp is connected to port 2 and is first put through an unshielded section and second through a shielded part of the HV cable. The ratio between both scattering parameters $S_{21}$ leads to the magnetic SE with:

$\mathrm{SE}_{\mathrm{H}, \text { meas }}=20 \log _{10}\left|\frac{\underline{S}_{21, \mathrm{ush}}}{\underline{S}_{21, \mathrm{sh}}}\right|$

Figure 8 proves that both numerical field simulation and laboratory measurements achieve well matching results in comparison to the models introduced in Sect. 3.1. The shielding behaviour can be described until approximately $1 \mathrm{MHz}$, since capacitive properties become relevant for even higher 


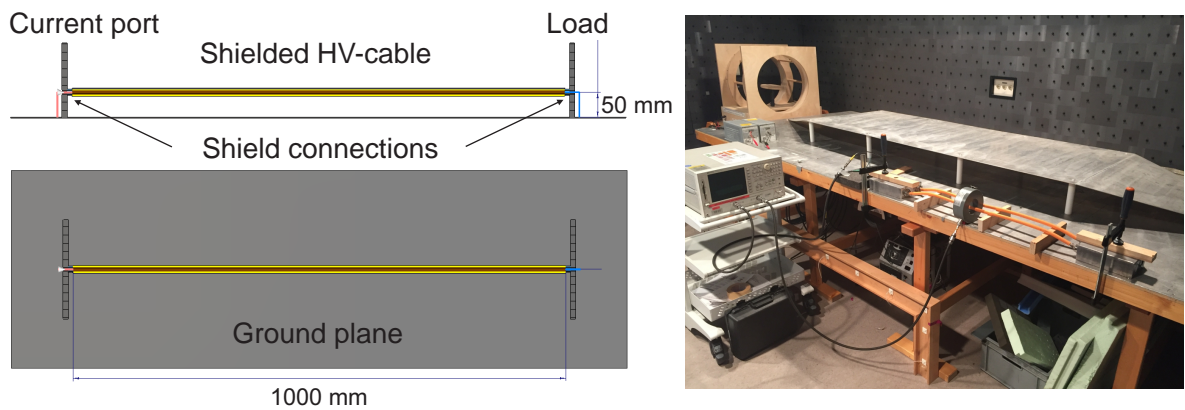

Figure 7. Left: 3-D Simulation Model (CM), right: measurement setup (DM).

frequencies. Having determined typical conditions for automotive HV systems, where $R_{\mathrm{gr}} \ll R_{\mathrm{sh}}$, magnetic $\mathrm{SE}$ values for CM and DM are nearly identical (see Eqs. 13 and 14). However, simulation and measurement data show a constant offset of about $2 \mathrm{~dB}$, when inductive influence becomes dominant. The reason behind are slightly different l.p.u. inductances following from Eqs. (15) and (16), which can be used to calculate the offset with $20 \log _{10}\left(L_{\mathrm{CM}, \text { in }} / L_{\mathrm{DM}, \text { in }}\right)$.

\subsection{Reference to automotive applications}

In general magnetic fields are attenuated by $20 \mathrm{~dB}$ per decade, not before the mutual inductance of the HV cable outweighs the DC-resistance of the shield. Low ohmic screens and screen transitions are therefore decisive to achieve a good shielding performance. At the moment OEMs request $3 \mathrm{~m} \Omega$ per metre screen and $9 \mathrm{~m} \Omega$ for each screen connection, based on investigations on the transfer impedance (Jacob, 2013). The method presented in this contribution can be used to review these EMC-requirements for electric vehicles in terms of magnetic fields.

Furthermore the ratio between mutual inductance and selfinductance of the shield plays a significant role. For ideal conditions they are equal and the magnetic field efficiency is continuously increasing with the frequency. In case of additional screen inductances, which are not part of the mutual inductive coupling, it converges to a specific maximum. In real application latter is the usual case, since HV connectors always have small but relevant parasitic inductances that restrict the shielding effect.

When refering to magnetic fields from CM currents, the influence of the ground also needs to be minded (see Eq. 13). For typical conditions, where the ground resistance is far lower than the shield resistance, the CM is basically similar to the DM. But there also might be constellations with high-ohmic ground paths. Even for no or low induced shield voltages, the current is now forced to flow via the shield path, leading to a significant SE even for DC and the very low frequency range. Transferring it to automotive power trains, this condition exemplary will be fulfilled, if HV-components are isolated from vehicle chassis, due to missing or bad protective earth (PE) connections. If there are major issues with
Table 3. Figure 9 legend.

\begin{tabular}{ll}
\hline Nr. & Description \\
\hline 1 & Dummy load (enclosed by metallic box) \\
2 & Power electronics (enclosed by metallic box) \\
3 & ANs for HV+ and HV- (enclosed by metallic box $)$ \\
4 & Copper plane \\
5 & Phase (AC) lines $(u, v, w)$ \\
6 & Traction (DC) lines (HV+/HV-) \\
7 & Control unit \\
8 & EMI Test Receiver \\
9 & HV DC Voltage Source \\
10 & Active loop antenna \\
\hline
\end{tabular}

CM currents, this behaviour can be used to improve magnetic shielding.

\section{Experimental validations on system level}

In the following derived findings of previous sections are validated in terms of magnetic field measurements on system level.

\subsection{Test setup}

The EMC tests are performed in an anechoic chamber, which enable radiated EMI measurements. In Fig. 9 the basic structure of the setup can be recognized. The centre box includes a prototypal automotive power electronics (EPF) from Continental with a switched-off internal DC/DC converter. Its native enclosure is connected to a surrounding metallic box, that is further grounded to a copper plane. To emulate the EMC behaviour of the electric motor a dummy load is used. It consists of three inductors with an inductance value of $100 \mu \mathrm{H}$ each. As parasitic capacitive coupling is very low for this configuration, three discrete capacitances $(2 \mathrm{nF})$ are added to obtain realistic conditions in terms of neutral-toground and phase-to-phase coupling. The dummy load is also surrounded by a conductive box. Two automotive artificial networks (ANs) are implemented to decouple the 

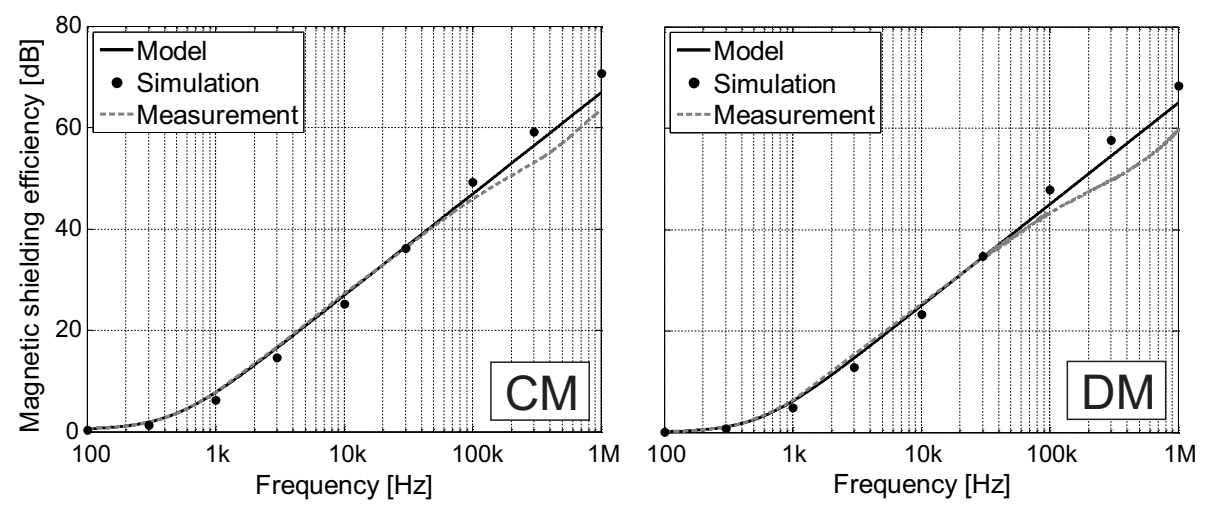

Figure 8. Magnetic shielding efficiency of typical HV cable configurations (left: CM; right: DM).
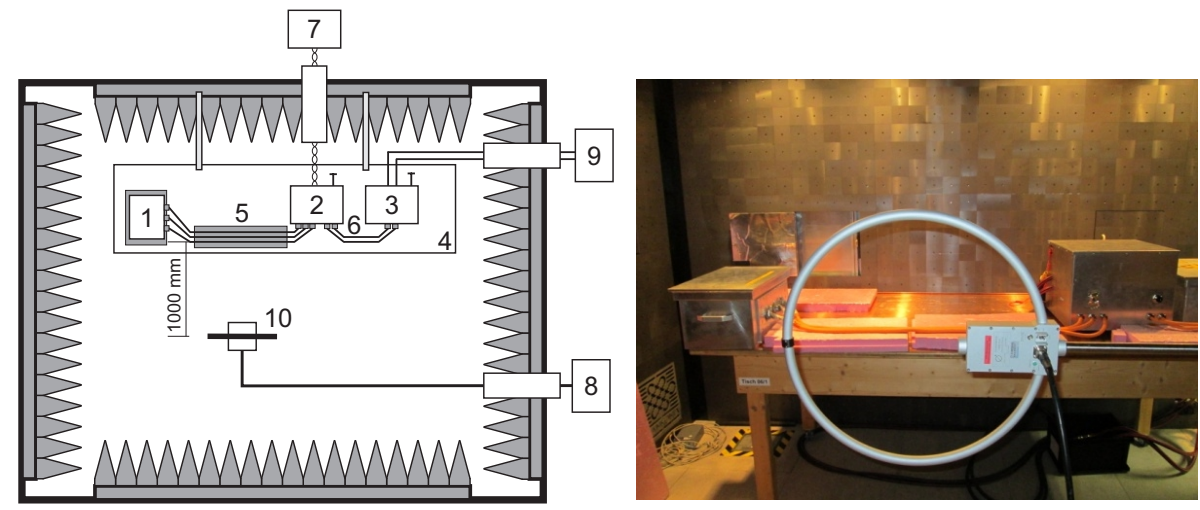

Figure 9. Test setup - H-field measurements of HV system demonstrator.

HV DC power supply from the power electronics. Detailed information about components insight the ANs are not required within the scope of the following investigations, as the primary focus is laid on the AC-side. Coroplast $\mathrm{HV}$ cables with Tyco connector systems are used for power transmission, where phase lines are $2 \mathrm{~m}$ and traction lines are $1 \mathrm{~m}$ long.

There are two operational modes used for experimental investigations below. First one is the so called "Idle Mode", where the operating load current is $0 \mathrm{~A}$ and the voltage switching characteristic equals a PWM with a constant duty cycle of $50 \%$ for all three phases. In an electric vehicle this mode correlates to the "Ready to Drive" state. Here, the inverter is already activated, but still causes no torque to run the electric engine. The second one is called the "Load Mode" with sinusoidal pulse-wide modulated phase-to-phase voltages to generate $50 \mathrm{~A}$ rotary current at a fundamental frequency of $400 \mathrm{~Hz}$. For both operational conditions the clock frequency is $10 \mathrm{kHz}$.

\subsection{Validation of the current spectrum}

Magnetic field measurements are performed with an unshielded cable configuration to correspond them with current spectra introduced in Sect. 2. The screens of the HV cables are still present, but disconnected at the load side. Because of the high-ohmic shield path, the magnetic SE is thus reduced to zero (see Eq. 12). Since currents are proportional to magnetic fields, there is a constant coupling factor over the observed frequency range, which is depend on the geometry of current paths and the distance as well as the polarisation of the loop antenna.

According to Fig. 10 the "Idle Mode" measurement follows the derivated CM current spectrum (see Sect. 2.1). This behaviour is expectable, because the in-phase PWM voltage switching is causing a set of pure CM harmonics. Considering "Load Mode", the spectral characteristic changes and a significant inductive influence from the dummy coils can be observed for lower frequencies. Next to the phaseto-neutral voltages, there are now additional phase-to-phase voltages and therefore respective DM currents (see Sect. 2.2). At approximately $2.5 \mathrm{MHz}$ a resonance point is shaped for both paths, where the current is only restricted by the DCresistance of the overall path. An EMC requirement for a country-specific type approval is indicated by a threshold according to GB/T 18387 (2008). In reference to these unshielded magnetic field measurements, the threshold is widely exceeded. 


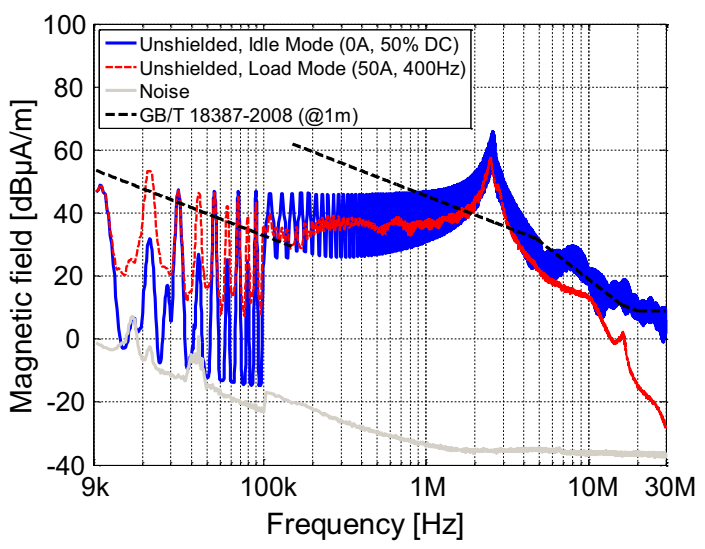

Figure 10. Experimental results to validate current spectra.

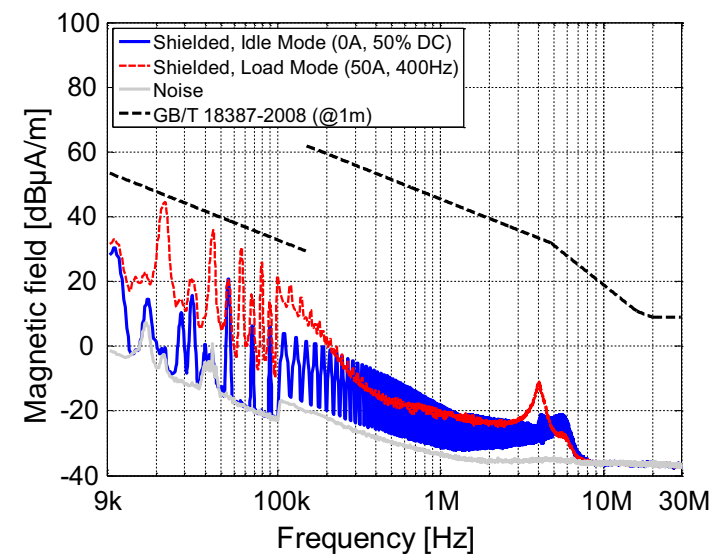

Figure 11. Experimental results to validate magnetic shielding efficiency.

\subsection{Validation of magnetic shielding efficiency}

In Figure 11 magnetic fields are measured for the shielded situation with cable screens, connected at both sides to the metallic enclosures. Hence, there is a magnetic shielding effect, due to the compensating induced shield currents described in Sect. 3. When comparing unshielded and shielded case, the magnetic SE is approximately $20 \mathrm{~dB}$ at $10 \mathrm{kHz}$ (clock frequency). Like expected it increases with $20 \mathrm{~dB}$ per decade. Remembering that CM and DM currents are simultaneously present in the "Load Mode", it is verified that respective magnetic fields are attenuated in the same way. Considering again the magnetic field threshold, EMC requirements for the shielded environment are now complied. However, for the loaded operating condition still critical magnetic fields are measured from EMC point of view. They can be directly referred to symmetrical current ripples and thus needs to be focused on, when designing HV topologies.

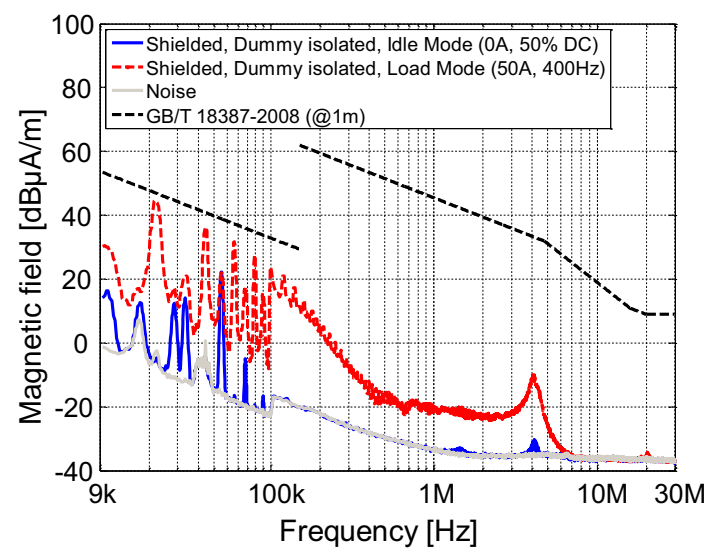

Figure 12. Experimental results to validate $\mathrm{CM}$ and DM current paths.

\subsection{Validation of current paths}

It might be relevant, whether either CM or DM currents are causing dominant magnetic fields. As they are superpositioned in the "Load Mode", the demonstrator must be slightly adapted to enable this kind of investigation. From Sect. 2.1 it is known, that CM currents are generated due to the capacitive coupled reference system, which is represented by the copper plane and cable screens in the test setup. If the copper plane is now isolated from the dummy load box, the return path is restricted to the screens. An ideal condition like in coaxial transmission lines is forced, where the inner wire equals the inverted shield current. Magnetic fields compensate and the shielding efficiency is high, even for DC and the very low frequency range (see Eq. 13). This effect is only related to CM currents, whereas magnetic fields from DM currents are attenuated like before (see Eq. 14).

In Fig. 12 respective measurements with isolated dummy boxes for shielded cables are displayed. As expected nearly no magnetic fields can be measured in the "Idle Mode", because of a set of pure CM current harmonics. Since there are always systemic asymmetries or other coupling paths like the DC-network, some minor current harmonics are left. Observing now the "Load Mode", the magnetic spectrum stays the same, although the CM part is completely removed. Hence, the loaded operating condition is dominated by magnetic fields caused by DM currents over the entire frequency range.

\section{Conclusions}

This contribution pointed out, that EMC-concepts for $\mathrm{HV}$ topologies of electric vehicles require the consideration of parasitic harmonic currents in the scope of magnetic fields. An appropriate countermeasure is to decrease parasitic Xand Y-capacitances distributed in electric motors and power electronics. Since it will never be possible to reduce them 
to zero and additional currents with inductive origins are present, the routing of $\mathrm{HV}$ cables gets a crucial role.

The introduced current analysis revealed the decisive current paths, which must be held geometrically small to minimize inductive coupling. For the CM case HV cables need to be positioned as near as possible to their reference system, which in general is the chassis of the vehicle. In order to reduce the area of DM current loops the single HV lines must lie as close as possible to each other. Especially in the region of the connectors, where cables are often spread, this requirement is difficult to follow. The use of sum shields and consequently less connectors might be advantageous in this context.

The magnetic shielding efficiency can be improved with low ohmic and low inductive screen connections. Particularly for low frequencies from DC to $100 \mathrm{kHz}$ it was demonstrated that attenuation is not sufficient to reduce magnetic fields far below the limit values. Adequate cable routing and elimination of parasitics thus remain an integral component of related EMC-concepts. If unshielded cables are used, an enhancement of magnetic fields up to $60 \mathrm{~dB}$ must be expected. In this case a well considered filtering system at the AC- and DC-side of the HV system becomes unavoidable.

Acknowledgements. This contribution was developed within the scope of the project EM4EM (Electromagnetic Reliability of Electronic Systems for Electro Mobility - Subproject: Entwurfs- und EMZ-Messmethodik für EMZ-Analysen auf EV-Gesamtsystemebene) which is funded by the BMBF (Bundesministerium für Bildung und Forschung) under the grant no. 16M3092A. The responsibility for this publication is held by the authors only.

Edited by: M. Chandra

Reviewed by: two anonymous referees

\section{References}

Cacciato, M., Consoli, A., Scarcella, G., and Testa, A.: Reduction of Common-Mode Currents in PWM Inverter Motor Drives, IEEE T. Ind. Appl., 35, 469-476, 1999.

CST EM Studio 2014: Workflow \& Solver Overview, CST - Computer Simulation Technology AG, Darmstadt, Germany, 2014.

Feldhues, K., Diebig, M., and Frei, S.: Analysis of the Low Frequency Shielding Behavior of High Voltage Cables in Electric Vehicles, International Symposium on Electromagnetic Compatibility (EMC Europe 2014), Gothenburg, Sweden, 1-4 September 2014, 408-413, 2014.

GB/T 18387-2008: Limits and test methods of magnetic and electric field strength from electric vehicles Broadband $9 \mathrm{kHz}$ to $30 \mathrm{MHz}$, EMC norm, 2008.

Grandi, G., Casadei, D., and Reggiani, U.: Common- and Differential-Mode HF Current Components in AC Motors Supplied by Voltage Source Inverters, IEEE T. Power Electr., 19, 1624, 2004.

Hohloch, J., Tenbohlen, S., Köhler, W., Aidam, M., and Ludwig, A.: Measurement of Transfer Impedances of Components for Automotive High-Voltage Power Networks, 2012 International Symposium on Electromagnetic Compatibility (EMC EUROPE), Rome, Italy, 17-21 September 2012 , 1-6, doi:10.1109/EMCEurope.2012.6396708, 2012.

Jacob, F., Heyen J., Rinkleff T., and Golisch F.: EMVAnforderungen an das Hochvoltbordnetz, GMM-Fachbericht 77: EMV in der Kfz-Technik, VDE Verlag GmbH, Stuttgart, 2013 (in German).

Jettanasen C.: Influence of Power Shielded Cable and Ground on Distribution of Common Mode Currents Flowing in VariableSpeed AC Motor Drive Systems, 2010 Asia-Pacific International Symposium on Electromagnetic Compatibility, Beijing, China, 12-16 April 2010, 953-956, 2010.

Kempski, A., Smolenski, R., and Strzelecki, R.: Common Mode Current Paths and Their Modeling in PWM Inverter-Fed Drives, Power Electronics Specialists Annual IEEE Conference - PESC, Cairns, Australia, 23-27 June 2002, 1551-1556, 2002.

Kempski, A. and Smolenski, R.: Decomposition of EMI Noise into Common and Differential Modes in PWM Inverter Drive System, Electrical Power Quality and Utilisation, XII, 53-58, 2006.

Paul, C. R.: Introduction to electromagnetic compatibility, 2nd Edn., Hoboken, NJ, Wiley, 2006. 